\title{
High Efficiency Triple-Junction Solar Cells Employing Biomimetic Antireflective Structures
}

\author{
Meng-Yih Chiu ${ }^{\mathrm{a}}$, Chia-Hua Chang ${ }^{\mathrm{b}}$, Feng-Yu Chang ${ }^{\mathrm{a}}$, and Peichen $\mathrm{Yu}^{\mathrm{b}}$ \\ ${ }^{a}$ Department of Photonics and Display Institute; \\ ${ }^{\mathrm{b}}$ Department of Photonics and Institute of Electro-Optical Engineering, \\ National Chiao Tung University, Hsinchu 30010, Taiwan \\ E-Mail: yup@faculty.nctu.edu.tw
}

\begin{abstract}
In this work, we demonstrate a thorough device design, fabrication, characterization, and analysis of biomimetic antireflective structures implemented on a $\mathrm{Ga}_{0.5} \mathrm{In}_{0.5} \mathrm{P} / \mathrm{GaAs} / \mathrm{Ge}$ triple-junction solar cell. The sub-wavelength structures are fabricated on a silicon nitride passivation layer using polystyrene nanosphere lithography followed by anisotropic etching. The fabricated structures enhance optical transmission in the ultraviolet wavelength range, compared to a conventional single-layer antireflective coating (ARC). The transmission improvement contributes to the enhanced photocurrent, which is also verified by the external quantum efficiency characterization of fabricated solar cells. Under one-sun illumination, the short-circuit current of a cell with a biomimetic structures is enhanced by $24.1 \%$ and $2.2 \%$ due to much improved optical transmission and current matching, compared to cells without an ARC and with a conventional ARC, respectively. Further optimizations of the biomimetic structures including the periodicity and etching depth are conducted by performing comprehensive calculations based on a rigorous couple-wave analysis method.
\end{abstract}

Keywords: Subwavelength structures, Nanostructures, Photovoltaic

\section{INTRODUCTION}

III-V compound multi-junction solar cells dominate the niche market of concentrator photovoltaics and space applications due to their direct-bandgap absorption, high temperature resistance, and wide material selectivity [1-2]. Currently, monolithically-grown $\mathrm{Ga}_{0.5} \mathrm{In}_{0.5} \mathrm{P} / \mathrm{GaAs} / \mathrm{Ge}$ cell structures are reported solar cells with the highest certified power conversion efficiency $\sim 32 \%$ under AM1.5g one-sun illumination [3]. The mature epitaxial technology can also make multi-junction solar cells very competitive for the terrestrial market. By incorporating a low-cost concentrator, the cell area can be minimized, where the material cost of multi-junction cells can potentially be amortized by their high power conversion efficiency. As a consequence, III-V multi-junction solar cells also appear as a promising candidate that could satisfy the requirement of third-generation photovoltaics [4].

In high-performance triple-junction solar cells, the antireflective coating (ARC) that accounts for the salvation of more than $30 \%$ optical loss is often realized by multi-layer dielectric stacking. However, issues such as polarization dependency, limited angular and spectral responses, mechanical instability due to thermal strain, etc. hinder the multijunction solar cells with a conventional ARC from fully exploiting their broadband optical absorption. Recently, the introduction of biomimetic sub-wavelength structures (SWS) serving as the antireflective layer has offered new prospective in the suppression of Fresnel reflection [5-6]. Due to the spatially graded structural profile in a single layer, the SWS ARC exhibits not only broadband and omnidirectional antireflective characteristics, but also the polarization insensitivity [7-12]. Moreover, the SWS ARC is also environmentally robust and mechanically durable, making it particularly desirable for concentrator and space photovoltaics. However, present proposed nano-fabrication techniques for SWS ARCs could result in the severe front surface recombination, which is the major obstacle in applying to III-V solar cells due to their high absorption coefficients [13-14]. In this paper, we demonstrate the implementation of antireflective nanostructures for a $\mathrm{Ga}_{0.5} \mathrm{In}_{0.5} \mathrm{P} / \mathrm{GaAs} / \mathrm{Ge}$ triple-junction solar cell. The biomimetic structures are fabricated on the silicon nitride (SiNx) passivation layer using polystyrene nanosphere lithography followed by anisotropic etching. The technique provides a relatively simple, scalable, and cost-effective means to control the structural profile [10, 1521], while minimizing the surface recombination current arising from etching. Under one-sun illumination, the shortcircuit current of a cell with the SWS ARC is enhanced by $24.1 \%$ and $2.2 \%$ due to much improved optical transmission

Physics and Simulation of Optoelectronic Devices XIX, edited by Bernd Witzigmann,

Fritz Henneberger, Yasuhiko Arakawa, Alexandre Freundlich, Proc. of SPIE Vol. 7933,

$79330 \mathrm{~N} \cdot$ - ( $2011 \mathrm{SPIE} \cdot \mathrm{CCC}$ code: 0277-786X/11/\$18 · doi: 10.1117/12.876850

Proc. of SPIE Vol. $793379330 \mathrm{~N}-1$ 
and current matching, compared to cells without a ARC and with a conventional single-layer (SL) ARC, respectively. The improvement of photocurrent is also investigated by the external quantum efficiency (EQE) characterization of fabricated solar cells, which matches the analysis of reflectance spectroscopy. Although the corresponding power conversion efficiency is merely comparable with the SL ARC cell due to a slightly degraded open-circuit voltage, the angular response is significantly improved over large angles of incidence. Further optimizations of the SWS ARC including the periodicity and etching depth are made possible by performing comprehensive calculations based on a rigorous couple-wave analysis (RCWA) method. The optimization technique allows to boosting the cell performance via improving optical transmission and current mismatch issues commonly seen in multi-junction solar cells.

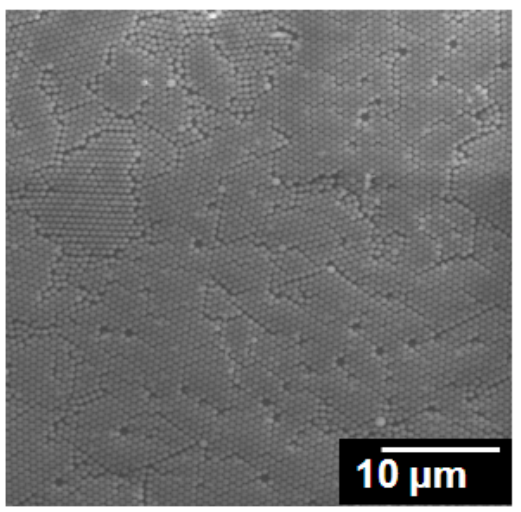

(a)

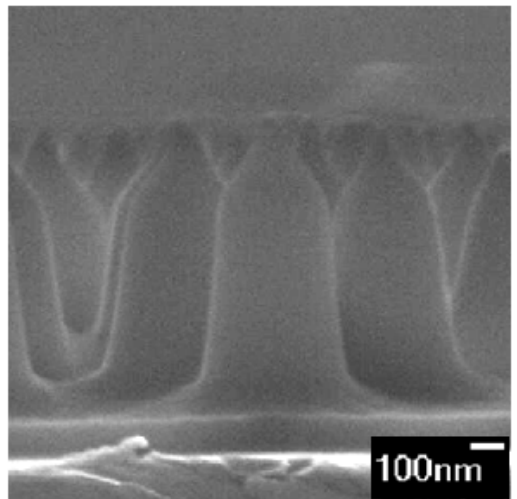

(b)

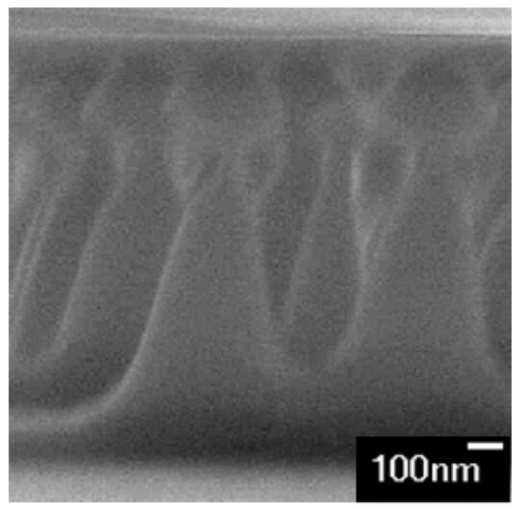

(c)

Fig. 1. (a) A large-area scanning electron micrograph of nearly-close-packed polystyrene nanospheres with a 600nm diameter deposited on $\mathrm{SiN}_{\mathrm{x}}$; (b) the cross-sectional view of $\mathrm{SiN}_{\mathrm{x}}$ biomimetic structures resulting from two-step and (c) one-step etching processes (residual nanospheres on top). A thin layer of $\operatorname{SiN}_{\mathrm{x}} \sim 100 \mathrm{~nm}$ thick is kept for passivation.

\section{INTRODUCTION}

Polystyrene (PS) nanosphere lithography is employed to fabricate SiNx-based SWSs on a triple-junction solar cell. First, $\mathrm{Ga}_{0.5} \mathrm{In}_{0.5} \mathrm{P} / \mathrm{GaAs} / \mathrm{Ge}$ cell structure was monolithically grown by metal-organic chemical vapor deposition (MOCVD) on a p-type germanium substrate. In the epi-structure, individual sub-cells were interconnected by highly-doped tunneling diodes, allowing band-to-band tunneling for the series output. At the beginning of cell processes, the GaAs ohmic layer was first lithographically defined by the selective etching of ammonia between the cap GaAs and $\mathrm{Al}_{0.5} \mathrm{In}_{0.5} \mathrm{P}$ window layer. A 1- $\mu$ m-thick silicon nitride $\left(\mathrm{SiN}_{\mathrm{x}}\right)$ was then deposited using plasma-enhanced chemical vapor deposition (PECVD). The SiNx has a refractive index of around 1.8 and a nearly-zero extinction coefficient characterized by an n\&k analyzer (n\&k Technology 1200). The SiNx film was subsequently treated with oxygen plasma to achieve a hydrophilic surface on which a mixture suspension of ethanol and polystyrene microspheres was spin coated. By tuning the degree of hydrophilicity, spinning speed, and the suspension concentration properly, a large area of nearly closepacked nanosphere monolayer arrays was obtained on a 4-inch wafer, as shown in Fig. 1 (a). Here, the PS spheres with a diameter of $600 \mathrm{~nm}$ serve as the etch mask for $\mathrm{SiN}_{\mathrm{x}}$. After steady air-drying for $15 \mathrm{~min}$., the samples with 1- $\mu \mathrm{m}$-thick SiNx were etched by an inductively-coupled-plasma reactive-ion-etching (ICP-RIE) system (OXFORD INSTRUMENTS, Plasmalab System 100) operated at $13.56 \mathrm{MHz}$ under a gas mixture of $\mathrm{CHF}_{3}$ and $\mathrm{O}_{2}$ through individual electronic mass flow controllers. The ratio of gas flow, chamber pressure, and etching time collectively control the sidewall profile, allowing the profile tuning of nanostructures. Figure 1(b) and 1(c) display two different etch profiles with an approximate etching depth of $900 \mathrm{~nm}$ using spheres with the same diameter of $600 \mathrm{~nm}$. The structure shown in Fig. 1(b) results from a two-step etching process and therefore shows an evident slope discontinuity. On the other hand, the side wall profile of Fig. 1(c) shows a smooth gradient which mimics the moth-eye structures. Hence the RIE condition of structures shown Fig. 1(c) is employed in the cell fabrication. We note that both in Fig.1 (b) and 1(c), a thin layer of $\mathrm{SiN}_{\mathrm{x}}, \sim 100 \mathrm{~nm}$ thick was kept intact for device passivation. After the fabrication of $\mathrm{SiN}_{\mathrm{x}} \mathrm{SWSs}$, an additional lithography step was applied to expose the ohmic GaAs layer by selectively etching the undesired SWSs. Finally, the front and back metal contacts were deposited by electron beam evaporation and annealed at $400^{\circ} \mathrm{C}$ for 35 sec. 
The fabricated SWS solar cells have a cell area of $0.5 \times 0.5 \mathrm{~cm}^{2}$ with a $10 \%$ metallic shadow ratio [22]. Moreover, cells without any AR treatment and with a conventional SL ARC, which contains a layer of 85-nm-thick SiN $\mathrm{x}_{\mathrm{x}}$, were also fabricated for comparison.
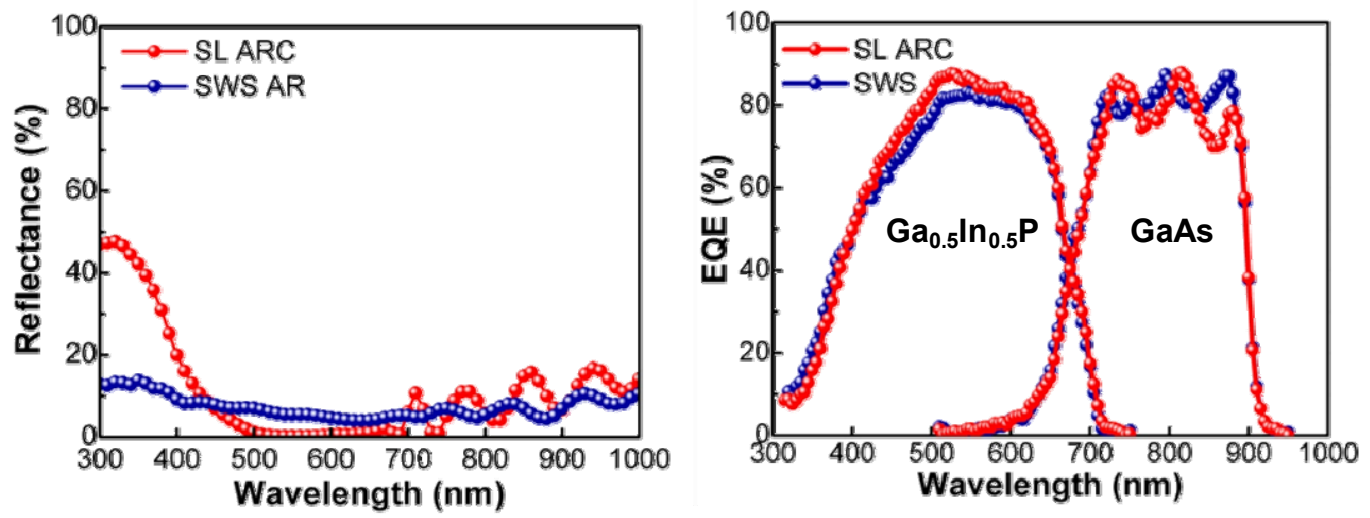

Fig. 2. (a) The measured reflectance spectra for cells with sub-wavelength structures (SWS) and a conventional singlelayer Antireflective Coating (SL ARC); (b) the measured external quantum efficiency (EQE) of the $\mathrm{Ga}_{0.5} \mathrm{In}_{0.5} \mathrm{P}$ top cell and the GaAs middle cell with SWS, SL ARC, and no ARC.

\section{INTRODUCTION}

\subsection{Photovoltaic characterization}

The reflectance spectra of cells with the SiNx SWSs and the SL ARC were measured using an UV/Visible/NIR spectrophotometer with a built-in integrating sphere (Hitachi U4100). The measured results are plotted in Fig. 2(a). Compared to the conventional ARC, the SWS ARC improves the optical transmission for ultraviolet/blue wavelengths, showing a relatively flat spectral response. Although the reflectance of the SWS ARC between 450 to $700 \mathrm{~nm}$ wavelength is relatively high, the AM1.5g-weighted reflectance of biomimetic SWSs is approximately $7.15 \%$ for the wavelength range of $300 \mathrm{~nm}$ to $1700 \mathrm{~nm}$, which is $\sim 2.5 \%$ lower than that of the SL ARC, $\sim 9.64 \%$. The suppression of Fresnel reflection contributes to the enhanced optical absorption, which is also reflected in the external quantum efficiency (EQE) characteristics of fabricated cells. Figure 2(b) shows the measured EQE results for the top and middle junctions for cells with the SWS, SL ARC, and without any ARC, respectively. As shown in Fig. 2(b), the EQE of the $\mathrm{Ga}_{0.5} \mathrm{In}_{0.5} \mathrm{P}$ top cell is indeed improved for $\lambda<450 \mathrm{~nm}$ and $\lambda>700 \mathrm{~nm}$ by using SWS, which agrees well with the reflectance measurement. As a result, the cell with the SWS ARC exhibits a higher short-circuit current, $\mathrm{J}_{\mathrm{sc}} \sim 11.62$ $\mathrm{mA} / \mathrm{cm}^{2}$ than that with the SL ARC, $\sim 11.37 \mathrm{~mA} / \mathrm{cm}^{2}$. The device current-voltage characteristics are summarized in Table 1. Overall, the short-circuit current of a cell with the SWS ARC is enhanced by $24.1 \%$ and $2.2 \%$ due to improved optical transmission and current matching, compared to cells without a ARC and with a SL ARC, respectively. The current-voltage characteristics are summerized in Table 1.

Table 1. AM 1.5g Current-Voltage Characteristics of Triple-Junction Solar Cells with Various Antireflective Coatings (ARCs)

\begin{tabular}{|cccc|}
\hline AR condition & No ARC & SLARC & SWS \\
\hline Voc (V) & 2.51 & 2.48 & 2.52 \\
Jsc (mA/cm²) & 9.36 & 11.37 & 11.62 \\
FF (\%) & 84.98 & 86.42 & 86.42 \\
IPCE (\%) & 19.93 & 24.41 & 25.26 \\
\hline
\end{tabular}




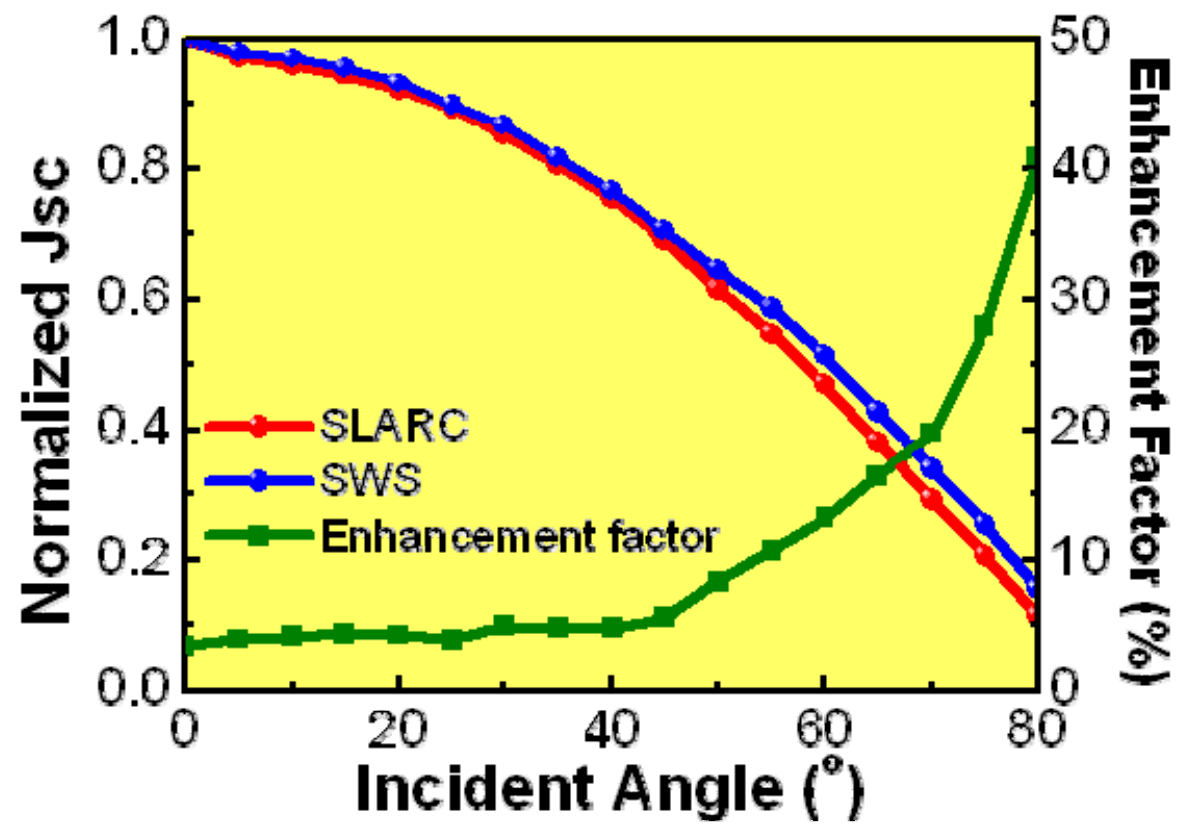

Fig. 3. The angular response of the short-circuit current for cells with SWSs and with a SL ARC. The calculated enhancement factor of the SWS cell demonstrates an improved angular response for AOI $>40^{\circ}$.

Moreover, the photocurrent enhancement was sustained and steadily increased over large angles of incidence. The angular response of the current-voltage characteristics was examined by mounting the cells on a customized rotatable chuck, where the photovoltaic characterization was performed under a simulated AM $1.5 \mathrm{~g}$ illumination source up to $80^{\circ}$. The angular responses of the short-circuit current densities, $\mathrm{J}_{\mathrm{sc}}$ are plotted in Fig. 3 for both cells with SWS and SL ARCs, which are normalized to those at normal incidence. As the angle of incidence increases to over $40^{\circ}$, the normalized Jsc of the cell with SWSs evidently decreases slower than that with a SL ARC. Further analyses reveal that the angular response of the normalized $\mathrm{J}_{\mathrm{sc}}$ for the SWS cell follows the cosine rule, which matches the descending trend of solar radiation at oblique incidence, i.e. the normal component of the incident photon flux density. The angular response shown in Fig. 3 confirms the omnidirectional antireflective characteristics of the biomimetic structures. Moreover, the enhancement of $\mathrm{J}_{\mathrm{sc}}$ is steadily improved over large angles of incidence (AOI), from less than $5 \%$ at $\mathrm{AOI}=0^{\circ}$ to more than $10 \%, \mathrm{AOI}>50^{\circ}$. In Fig. 3 , the enhancement factor is defined as

$$
E F(\%)=\left(\frac{J_{S c}(\theta)_{\mathrm{sWS}}}{J_{S C}(\theta)_{\mathrm{SLARC}}}-1\right) \times 100 \%
$$

where $\mathrm{J}_{\mathrm{SC}}(\theta)_{\mathrm{SwS}}$ and $\mathrm{J}_{\mathrm{SC}}(\theta)_{\text {SLARC }}$ represent the short-circuit current density at different angles of incidence for cells with the SWS and SL ARC, respectively.

\subsection{RCWA simulation}

The rigorous couple wave analysis (RCWA) approach has been employed to investigate the diffraction and transmission properties of nanoscale structures [23]. In this work, a commercial implementation of the three-dimensional RCWA (DIFFRACTMOD, Rsoft Corporation) is employed to first examine the consistence between the simulation and experiment, and to further optimize the SWSs. As shown in the inset of Fig. 4, the simulated structural profile of a unit cell consists of $7 \mathrm{x} 7 \mathrm{SiN}_{\mathrm{x}}$ SWSs in a hexagonal array. The side wall profile is approximated with a parabolic function to match the structures seen Fig. 1(c). Moreover, a variation of 10\% in the structural height was introduced to account for the fluctuation in the etching depth. Below the $\operatorname{SiN}_{\mathrm{x}}$ SWS layer include a thin $\mathrm{SiN}_{\mathrm{x}}$ passivation layer, $\sim 100 \mathrm{~nm}$ thick, the $\mathrm{Al}_{0.5} \mathrm{In}_{0.5} \mathrm{P}$ window layer, and the $\mathrm{Ga}_{0.5} \mathrm{In}_{0.5} \mathrm{P}$ top cell. The material dispersion of each layer was measured and taken into account for wavelengths between $300 \mathrm{~nm}$ and $1000 \mathrm{~nm}$. The electric field is set to be 45 -degree linearly polarized to mimic the solar radiation. As shown in Fig. 4, the simulated reflectance agrees well with the experimental data measured 
by the integrating sphere (shown in Fig. 2(b)) for wavelengths larger than 500nm. A slight discrepancy occurs at short wavelengths, possibly due to the mismatched material dispersion used for simulation. Further characterization on the complex refractive indices of $\mathrm{Ga}_{0.5} \mathrm{In}_{0.5} \mathrm{P}$ and $\mathrm{Al}_{0.5} \mathrm{In}_{0.5} \mathrm{P}$ layers is required to improve the accuracy.

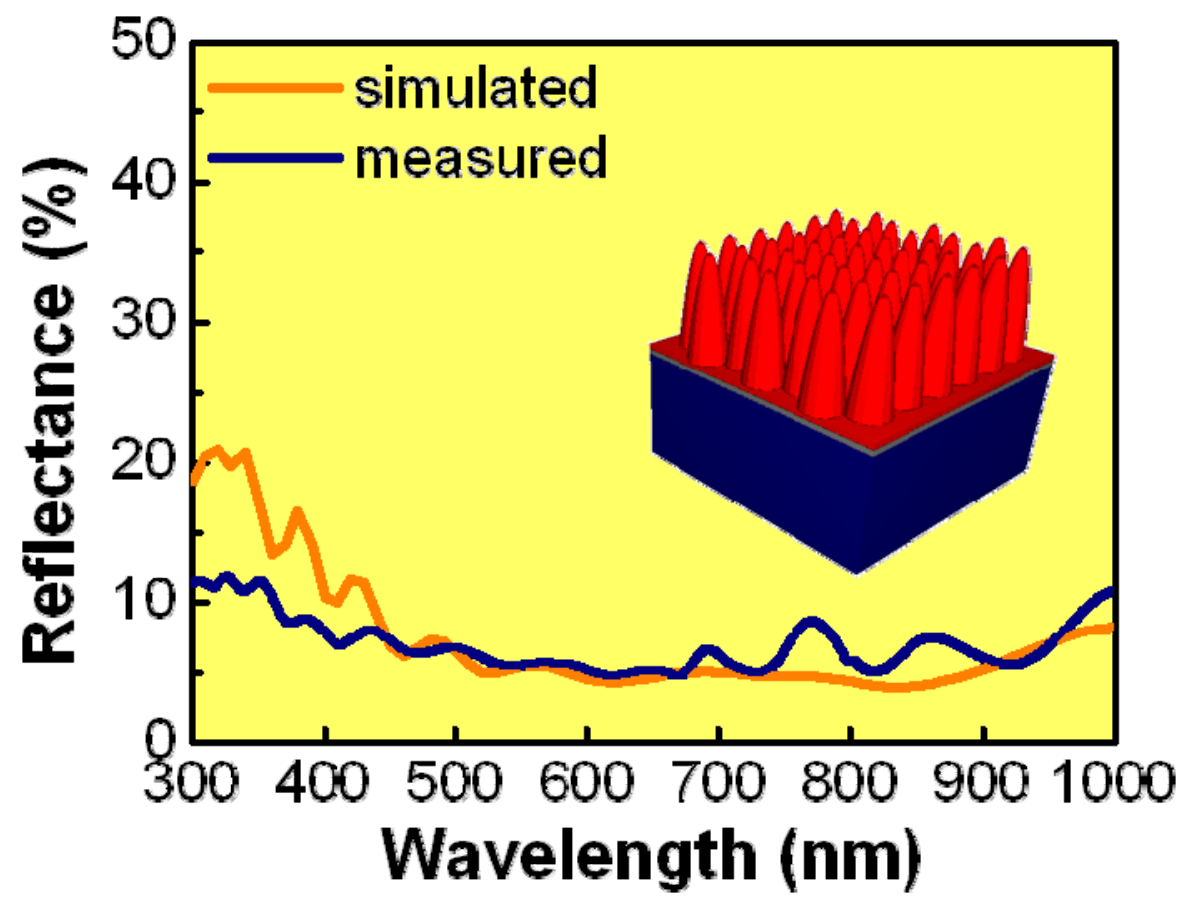

Fig. 4. The comparison between the calculated and the measured optical reflectance. The inset shows the simulated unit cell which comprises an array of $7 \mathrm{x} 7 \mathrm{SiN}_{\mathrm{x}}$ paraboloids, a thin $\mathrm{SiN}_{\mathrm{x}}$ passivation layer, and the top cell structures.

After confirming the validity of our simulation model for wavelengths larger than $450 \mathrm{~nm}$, we can optimize the optical reflectance as a function of periodicity, i.e. the nanosphere diameter, and the structural height, i.e. the etching depth. The calculations are performed at a fixed aspect ratio of 1.5 (height/periodicity=900 nm/600 nm) at which a set of stable fabrication parameters is guaranteed. The calculated equal-reflectance contours as a function of wavelength are plotted in Fig. 5(a) and 5(b) for the periodicity and height, respectively. As shown in Fig. 5(a), PS spheres with a large diameter reduce optical reflectance for long wavelengths. Moreover, figure 5(b) suggests that an etching depth of $600 \mathrm{~nm}$ is sufficient for the absorption enhancement for the top junction. However, the etching depth should be chosen based on the current matching of the top junction to other junctions. It is also feasible to lower the reflectance for diffident wavelength ranges by varying the etching depth. Figure 5(a) and 5(b) indicate that the biomimetic structures allow the broadband antireflection engineering tailored for specific spectral response of various tandem cells.

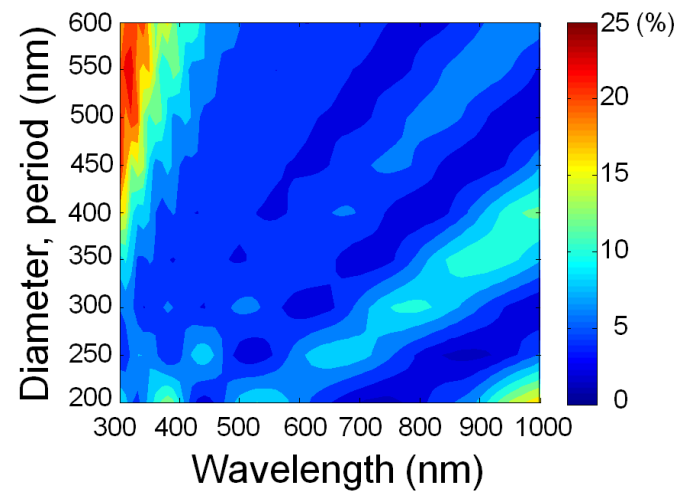

(a)

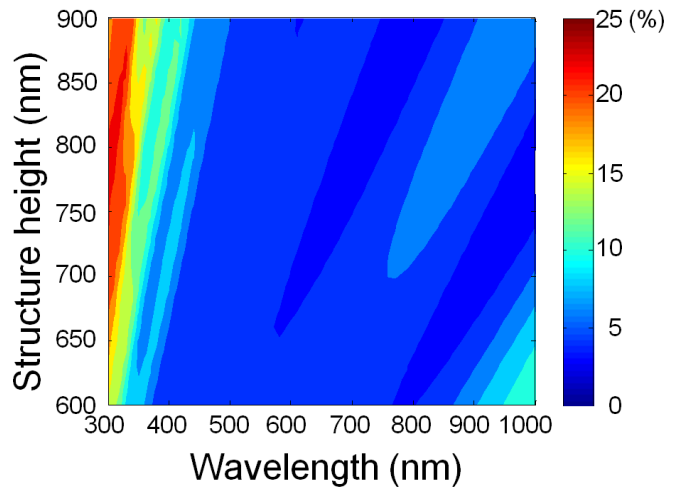

(b)

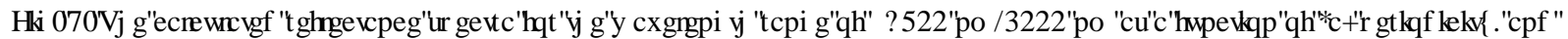

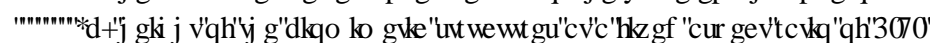




\section{INTRODUCTION}

In conclusion, we have successfully fabricated $\mathrm{SiN}_{\mathrm{x}}$-based biomimetic antireflective structures for a $\mathrm{Ga}_{0.5} \mathrm{In}_{0.5} \mathrm{P} / \mathrm{GaAs} / \mathrm{Ge}$ triple-junction solar cell employing the polystyrene nanosphere lithography. The side-wall profiles of sub-wavelength structures can be controlled by adjusting etch parameters. The reflectance spectroscopy and external quantum efficiency measurements confirm the improved optical absorption in the ultraviolet/blue wavelengths. Under one-sun AM1.5g illumination, the short-circuit current of a cell with biomimetic structures is enhanced, while the enhancement is sustained and amplified over a large angle of incidence. Calculations based on a rigorous coupled-wave analysis not only verify the broadband antireflection consistent with the measured data, but also serve as an optimization tool for the implementation of biomimetic antireflective structures in multi-junction solar cells.

\section{ACKNOWLEDGMENTS}

The authors thank Prof. H. C. Kuo at the Department of Photonics, National Chiao Tung University in Taiwan for technical support. This work is founded by National Science Council in Taiwan under grant number 96-2221-E-009-095MY3 and 97-2120-M-006-009.

\section{REFERENCE}

[1] Olson, J. M., Friedman, D. J. and Kurtz, S., [Handbook of Photovoltaic Science and Engineering], Academic, Orlando, Fla., (2003).

[2] Yamaguchi, M., "III-V compound multi-junction solar cells: present and future," Solar Energy Mat. And Solar Cells 75, 261-269 (2003).

[3] Green, M. A., Emery, K., Hishikawa. Y. and Warta, W., “Solar cell efficiency tables (version 34) ,” Prog. Photovolt. Res. Appl. 17, 320-326 (2009).

[4] Conibeer, G., “Third-generation photovoltaics,” Materials Today 10, $42-50$ (2007).

[5] Stavenga, D. G., Foletti, S., Palasantzas G., and Arikawa, K., "Light on the moth-eye corneal nipple array of butterflies,” Proc. R. Soc. B. 273, 661-667 (2006).

[6] Parker, A. R., Townley, H. E., “ Biomimetics of photonic nanostructures,” Nature Nanotechnology 2, 347-353 (2007).

[7] Huang, Y. F., Chattopadhyay, S., Jen, Y. J., Peng, C. Y., Liu, T. A., Hsu, Y. K., Pan, C. L., Lo, H. C., Hsu, C. H., Chang, Y. H., Lee, C. S., Chen K. H., and Chen L. C., "Improved broadband and quasi-omnidirectional antireflection properties with biomimetic silicon nanostructures,” Nature Nanotech. 2, 770-774 (2007).

[8] Hobbs, D. S., MacLeod, B. D., and Riccobono, J. R., "Update on the development of high performance antireflecting surface relief micro-structures,” Proc. SPIE 6545, 65450Y-1-14 (2007).

[9] Sai, H., Kanamori, Y., Arafune, K., Ohshita, Y., Yamaguchi, M., "Light trapping effect of submicron surface textures in crystalline Si solar cells," Progress in Photovoltaics: Research and Applications 15, 415-423 (2007).

[10] Chiu, C. H., Yu, P., Kuo, H. C., Chen, C. C., Lu, T.C., Wang, S. C., Hsu, S. H., Cheng, Y. J., and Chang, Y. C., "Broadband and omnidirectional antireflection employing disordered GaN nanopillars" Opt. Exp., 16, 87488754 (2008).

[11] Kanamori, Y., Sasaki, M., and Hane, K., "Broadband antireflection gratings fabricated upon silicon substrates," Opt. Lett., 24, 1422-1424 (1999).

[12] Lee, Y.-J., Ruby, D. S., Peters, D. W., McKenzie, B. B., and Hsu, J. W. P., "ZnO Nanostructures as efficient antireflection layers in solar cells,” Nano Lett., 8, 1501-1505 (2008).

[13] Peng, K., Xu, Y., Wu, Y., Yan, Y., Lee, S. T., and Zhu, J., “Aligned Single-Crystalline Si Nanowire Arrays for Photovoltaic Applications,” Small 1, 1062-1067 (2005).

[14] Garnett, E., Yang, P., “Silicon Nanowire Radial p-n Junction Solar Cells,” J. Am. Chem. Soc., 130, 9224-9225 (2008). 
[15] Wang, B., Zhao, W., Chen, A., Chu, S.-J., "Formation of nanoimprinting mould through use of nanosphere lithography,” Journal of Crystal Growth, 288, 200-204 (2006).

[16] Chen, H. L., Chuang, S. Y., Lin, C. H., and Li, Y. H., "Using colloidal lithography to fabricate and optimize sub-wavelength pyramidal and honeycomb structures in solar cells," Opt. Exp., 22, 14793-14803 (2007).

[17] Sun, C.H., Jiang, P., Jiang. B., "Broadband moth-eye antireflection coatings on silicon,” Appl. Phys. Lett., 92, 061112-1-061112-3 (2008).

[18]Hsu, C.-M., Connor, S. T., Tang, M. X., and Cui, Y., "Wafer-scale silicon nanopillars and nanocones by Langmuir-Blodgett assembly and etching,” Appl. Phys. Lett., 93, 133109-1-133109-3 (2008).

[19] Chan, C. H., Hou, C. H., Tseng, S. Z., Chen, T. J., Chien, H.T., Hsiao, F. L., Lee, C. C., Tsai, Y. L., Chen, C.C., "Improved output power of GaN-based light-emitting diodes grown on a nanopatterned sapphire substrate," Appl. Phys. Lett., 95, 011110-1-011110-3, (2009).

[20] Li, Y., Zhang, J., Zhu, S., Dong, H., Jia F., Wang, Z., Sun, Z., Zhang, L., Li, Y., Li, H., Xu, W., Yang, B., "Biomimetic Surfaces for High-Performance Optics," Adv. Mat. 21, 4731-4734, (2009).

[21] Chen, Q., Hubbard, G., Shields, A., Liu, C., Allsopp, D. W. E., Wang, W. N., Abbott, S.,” Broadband moth-eye antireflection coatings fabricated by low-cost nanoimprinting,” Appl. Phys. Lett., 94, 263118-1-263118-3, (2009).

[22] Emery, K., "Measurement and characterization of solar cells and modules ," in Handbook of Photovoltaic Science and Engineering, Antonio Luque, Steven Hegedus, ed. (Academic, Orlando, Fla., 2003).

[23] Nevière M., and Popov, E., [Light Propagation in Periodic Media], Marcel Dekker, New York, (2003). 